\title{
Research on Terrain Reconstruction of Twin Sequence Images in Lunar Exploration
}

\author{
Wang Wei, Zhao Wenbin and Zhao Zhengxu \\ School of Information Science and Technology \\ Shijiazhuang Tiedao University \\ Shijiazhuang, Hebei, China \\ wangwei@stdu.edu.cn_zhaowb19851015@gmail.com_zhaozx@stdu.edu.cn
}

\begin{abstract}
The detectors are an important tool in deep space exploration; they can provide a large number of images which contain the ground environment information of celestial body. Bythree-dimensional reconstruction, terrain information can be extracted from these images, such as elevation data and texture data. This paper focuses on an adaptive terrain reconstruction for sequence image. A method, which calculate Binocular geometric model with matching measures of matching points and random sample consensus algorithm, is proposed. On the basic of this method, a densepoints matching method based on epipolar and homography constraints is presented. Besides the selection method of reconstruction image and the terrain reconstruction flow are discussed. The proposed adaptive terrain reconstruction satisfies the automation and real time requirement of some terrain reconstruction task, such as landing filed selection in detector landing, and is experiment and applied into sequence image of detector ChangE III's landing camera, so as to ensure the safety of detector landing.
\end{abstract}

Keywords: terrain reconstruction, lunar exploration, twin sequence image, image matching, detector, landing

\section{Introduction}

With the continuous development of space technology, computer technology, remote sensing technology, the method and ability of spatial information acquisition are growing, meanwhile the acquired data is increasing. The acquired data contains a large number of sequence landform images. By three-dimensional reconstruction technology, terrain information can be extracted from sequence landform images, such as elevation data and texture data. The extracted information helps people cognize spatial terrain environment, and play an important role in deep space exploration.

In order to apply terrain reconstruction technology into sequence images in deep space exploration, this paper focuses on adaptive terrain reconstruction for sequence image. A method, which uses matching measures of image feature points and random sample consensus algorithm to linearly calculate the parameter of two views relative geometric relation, is proposed. On the basic of calculation, a dense points matching method based on epipolar and homography constraint is presented, it searches in small range to increases speed, and improves the reliability and precision of automatic matching. On automation and real-time performance, reconstruction image selecting method and terrain reconstruction flow 
are discussed. To determine detector ChangE III's landing field, terrain reconstruction algorithm is applied and experimented into the sequence image of landing camera.

\section{Related Work}

In 1970s, Marr, Barrow and Tenenbaum, et al., created a set of visual computing theory, which extracts three-dimensional structure information from image [1]. By mid 1980s, Stereo vision has been researched and applied widely in many fields. Dhond and Aggarwal Analyzed the status of stereo vision in 1980s, introduced A large number of image matching method, and adopted trinocular stereo constraints to reduce ambiguity of stereo matching on the basis of hierarchy process concept [2].

After 1990s, many aspect of stereo vision research began to mature and made important overall progress in many areas, such as area matching, feature matching, occlusion handling, several cameras stereo vision, real-time algorithm and so on. Brown, Burschka and Hager introduced the progress of computer 3D vision research during 10 years since 1993, and discussed feature matching, occlusion handling and real-time algorithm [3].

Scharstein and Szeliski compared the performance of several common algorithms [4]. Schmid and Zisserman discussed Automatic straight line matching method and multi view reconstruction method [5]. Rother, et al., presented a linear multiple view reconstruction method, which can compute camera parameter [6]. Hartley and Faugeras, et al., gave many multiple view reconstruction algorithms [7, 8].

\section{Featrue Points Matching Algorithm}

For terrain reconstruction of sequence images in deep space exploration, feature points' matching is a crucial step. This section presented the basic matrix of stereo vision is calculated by matching measure of feature points' initial matching result and RANSAC algorithm, and according to accurate matching result which is optimized by calculated basic matrix, the dense points matching is obtained by homography and epipolar constraint.

\subsection{Accurate Matching Based on Basic Matrix}

At present, the extraction and matching of feature points and the calculating of basic matrix are independent of one another $[9,10]$. This paper gives a method of basic matrix calculation which uses the matching measure and RANSAC algorithm to calculate basic matrix and obtain accurate feature points matching. This method use the matching measure to normalize the set of matching points, which is used to calculate the basic matrix in RANSAC algorithm.

1) Matching Measure

In matching points, different matching points have different matching precision. Matching measure can be used to analyze the matching precision. The function of matching measure is as follow:

(1)

Where is correlation coefficient which is related to matching methods, for example, the method of grayscale correlation defines, the method of distance correlation defines. If the value of matching measure is bigger, the corresponding matching points are more accurate.

By the matching measure, the function of matching points' weight is defined as follow:

(2) 
The function of matching points' weight is used to normalize the set of matching points.

2) RANSAC Algorithm

RANSAC algorithm is proposed by Fischler and Bolles, which calculate the parameter of mathematical model with sample data set to obtain valid data set. With RANSAC algorithm, the minimum sets are select repeatedly from matching points set to calculate each basic matrix, then the consistent sets which are selected from the minimum sets are used to calculate basic matrix. With RANSAC algorithm, Specific steps of basic matrix calculating are as follows:

a) Feature points are extracted with Forstner algorithm, and are matching initially with Least Squares Matching method based on Scale Invariant Feature. Then matching measure of matching points is calculated.

b) For uniform discrete distributions, eight matching points, whose distance satisfies the requirement of threshold value, are selected randomly from initial matching result.

c) Basic matrix is calculated with selected matching points which are normalized by matching measure.

d) With the formula which is as follow, the distance between matching points and model is calculated. If calculated distances satisfy the requirement of threshold value, the corresponding matching points is considered as accurate matching points.

e) Repeat above steps $2-4$, all accurate matching points are recorded.

f) The recorded matching points are normalized, then used to calculate basic matrix.

\subsection{Dense Points matching based on Homography and Epipolar Constraint}

For the matching points' number requirement of three-dimensional reconstruction, dense points are matching lonely with Homography or Epipolar constraint [11-13]. This paper gives a dense points matching method based on Homography and Epipolar constraint. The process of dense points matching method based on Homography and Epipolar constraint is as follow:

a) Feature points are extracted with Forstner algorithm, and are matching initially with Least Squares Matching method based on Scale Invariant Feature. Then matching measure of matching points is calculated.

b) Accurate matching points are selected with matching measure and RANSAC algorithm, and used to calculate basic matrix.

c) The matching points set in any unmatching point's neighborhood are selected.

d) Homography matrix is calculated repeatedly with four matching points group selected from matching points set. If calculated homography matrix satisfies the compatibility relation between basic matrix and homography matrix, the corresponding matching points group is recorded.

e) The matching points group, which are the nearest to unmatching point, are selected from record matching points group, and used to calculated the corresponding point of unmatching point.

f) The calculated point is optimized by Epipolar constraint.

The dense points matching method based on Homography and Epipolar constraint reduces retrieval scope, so as to improve accuracy and efficiency of dense points matching.

\section{Reconstruction Image Selection Method}

For terrain construction of sequence image, construction images are selected from sequence image, which has continuity on time and overlap on region. The selected 
reconstruction images must satisfy the intersection condition, which include overlapping ratio and intersection angle between images.

Let is the height of imaging, is the focal length of camera, is the vision angle of camera, is the distance between imaging positions, and is the length of images' intersection scope in movement direction, as shown in Figure 1.
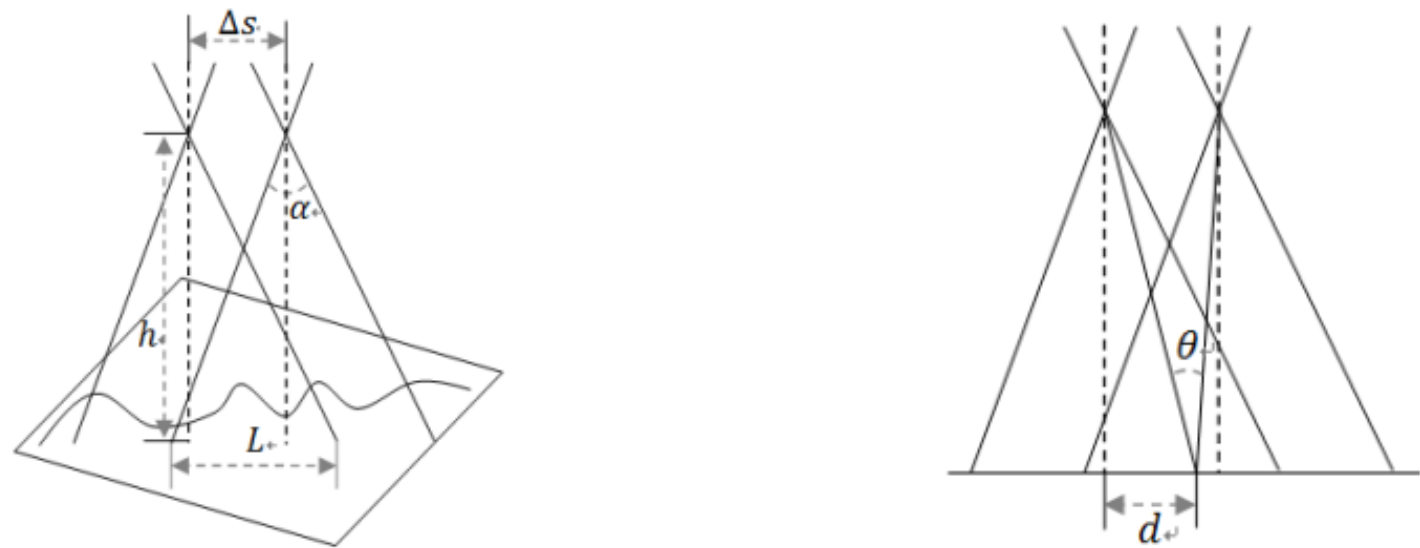

Figure 1. The Geometric Relation between Sequence Images

1) Overlapping Ratio

Let is the overlapping ratio between sequence images, which is the ratio of overlapping scope in corresponding image. The requirement of image's overlapping ratio satisfies, the overlapping ratio set by experience.

The length of images' intersection scope in movement direction and the distance between imaging positions are satisfied as follow:

(4)

The overlapping ratio and the length of images' intersection scope in movement direction are satisfied as follow:

(5)

The distance between imaging position and the overlapping ratio are satisfied as follow:

(6)

According to the minimum overlapping ratio, the maximum distance between imaging position is as follow:

2) Intersection Angle

Let is the intersection angle between sequence images, which is an angle formed by the positions of imaging and the position in overlapping area. The requirement of image's intersection angle satisfies, the intersection angle set by experience.

The intersection angle and the distance between imaging positions are satisfied as follow:

(8)

The minimum intersection angle is as follow:

(9)

Where

The minimum intersection angle and the distance between imaging positions are satisfied as follow: 
(10)

According to the minimum intersection angle, the minimum distance between imaging position is as follow:

(11)

3) Intersection Condition

According to overlapping ratio and intersection angle, the intersection condition of reconstruction images is satisfied, so the selection of reconstruction image is satisfied as follow:

(12)

The distance between imaging positions should be select as large as possible, as follow:

\section{Adaptive Terrain Reconstruction Flow}

For the time continuity and region overlap of sequence image, terrain reconstruction algorithm flow is proposed on image feature points' extraction, matching and stereo vision's basic matrix computation, as shown on Figure 2.

a) This algorithm flow's input include sequence image, the position and posture of imaging, the internal and external parameters of camera, etc. the sequence image is selected by analyzing imaging's movement distance.

b) The extraction of image feature points uses Forstner algorithm, which selects the extreme point of Gray level and gradient change in image's local scope as feature point.

c) The initial matching of image feature points uses Least Squares Matching based on Scale Invariant Feature.

d) Based on the matching measure of the initial matching result, the basic matrix of stereo vision is computed by RANSAC algorithm.

e) The initial matching result is optimized by computed basic matrix.

f) The dense points is matched by homography and epipolar constraint.

g) The essential matrix is calculated by the basic matrix of stereo vision, the internal and external parameters of camera, so that dense points are 3 -dimentional constructed.

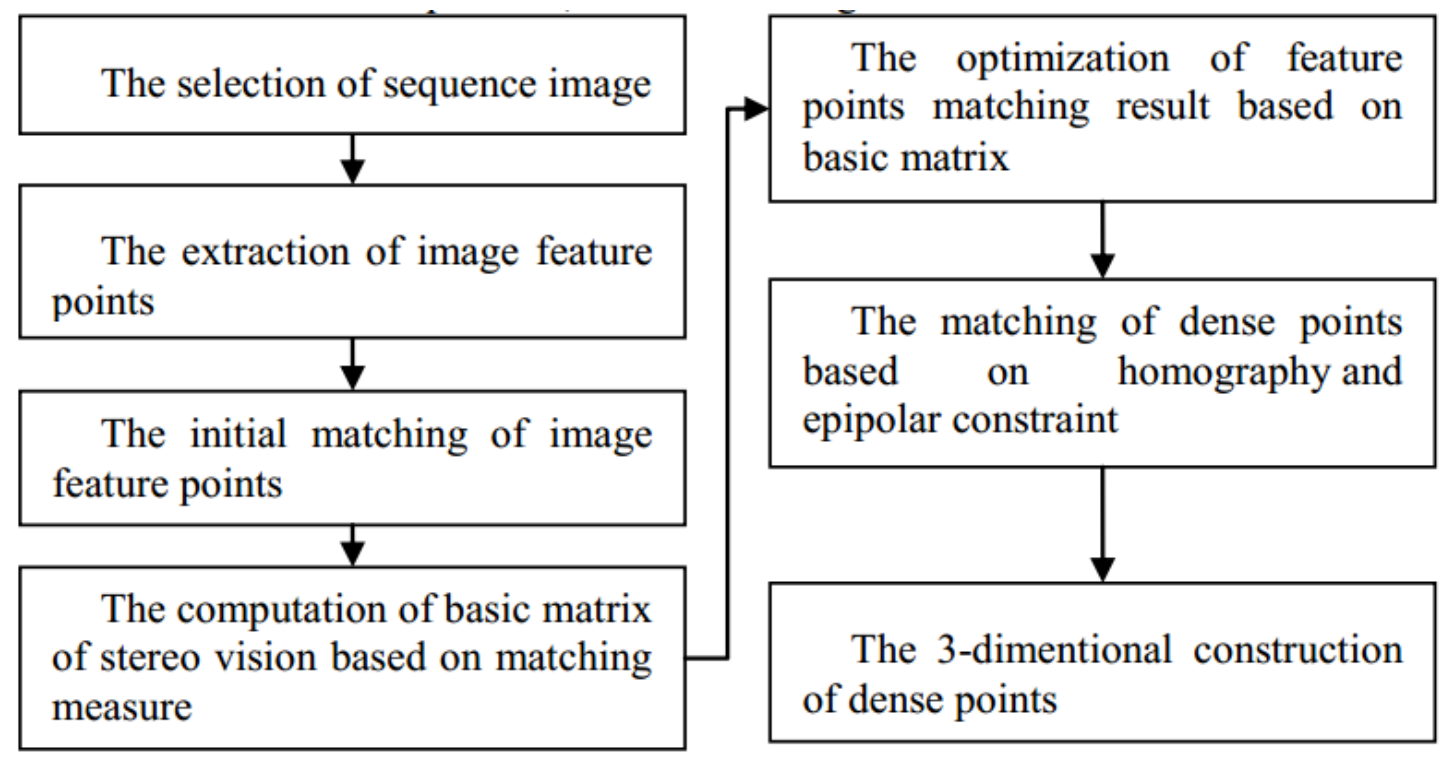




\section{Figure 2. Terrain Reconstruction Algorithm Flow of Sequence Image}

\section{Experiment and Application}

Terrain reconstruction algorithm is applied into the experimental image of detector ChangE III's landing camera, as shown in Figure 3. Figure 3(a) is the extraction and initial matching result of image feature points, Figure $3(\mathrm{~b})$ is the accurate matching result if image feature points, Figure 3(c) is the matching result of dense points for image feature points, Figure 3(d) is the triangle grid of terrain reconstruction result's elevation data, Figure 3(e) is the visualization of terrain reconstruction result's elevation data and texture data.

This application is experimented on PC, which has Intel $3.2 \mathrm{GHz}$ Xeon CPU and 16GB DDR3 memory, meanwhile the threshold of dense points number set 300 . The average time of terrain reconstruction which is from feature points' extraction and matching to $3 \mathrm{D}$ reconstruction is above $368 \mathrm{~ms}$, so this terrain reconstruction algorithm is satisfied the real-time requirement of terrain reconstruction for landing camera sequence image.

In addition, In the Figure 3(a), Figure 3(b) and Figure 3(c), left photographs are shot by the left camera, right photographs are shot by the right camera.

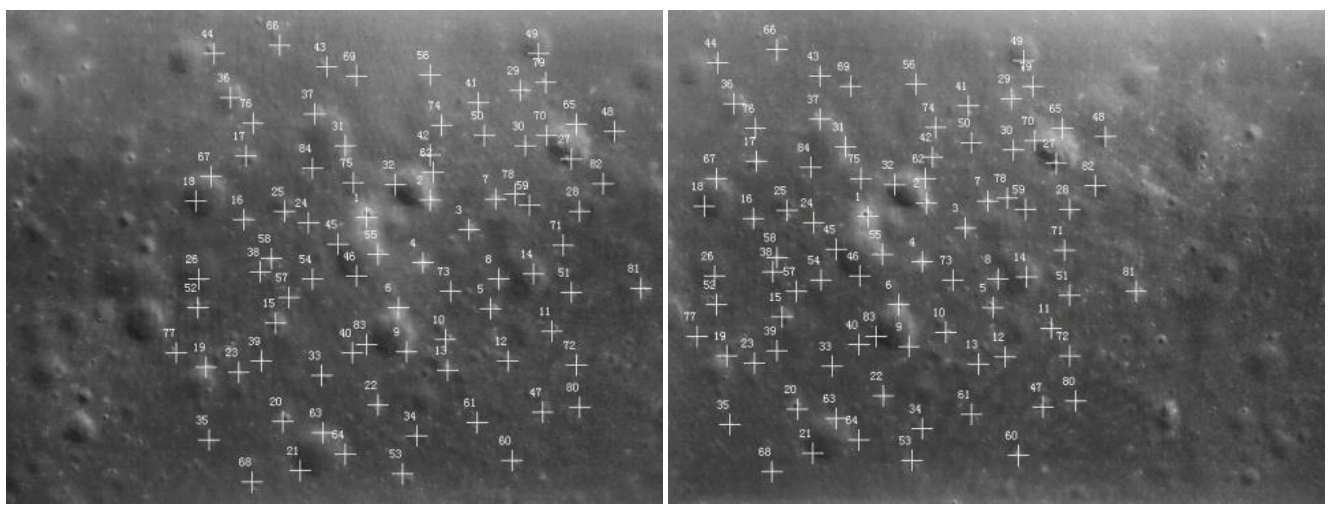

(a)The extraction and initial matching result of image feature points
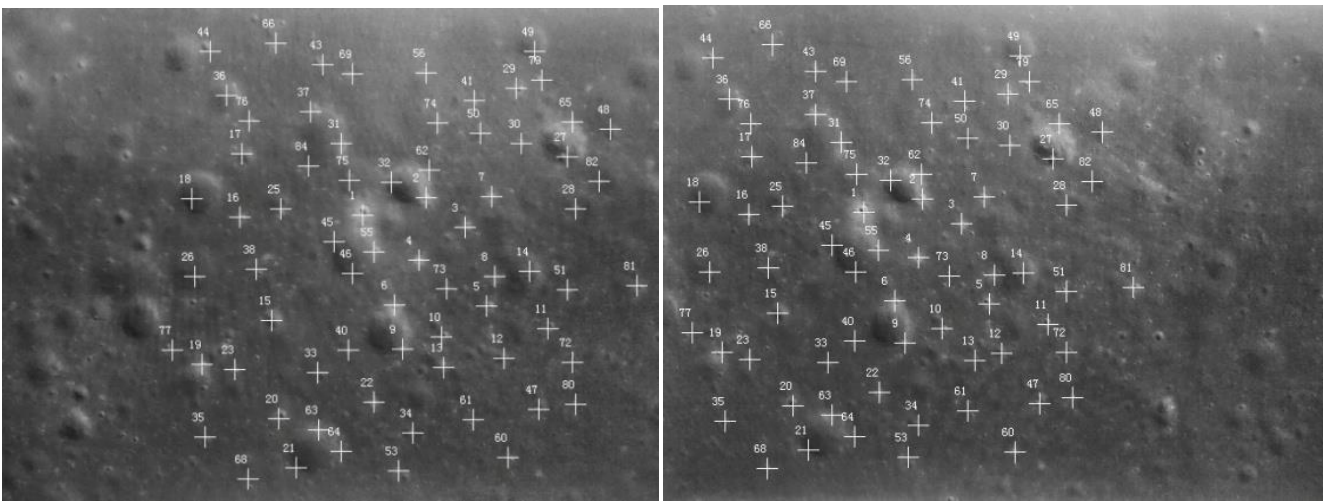

(b) The accurate matching result if image feature points 


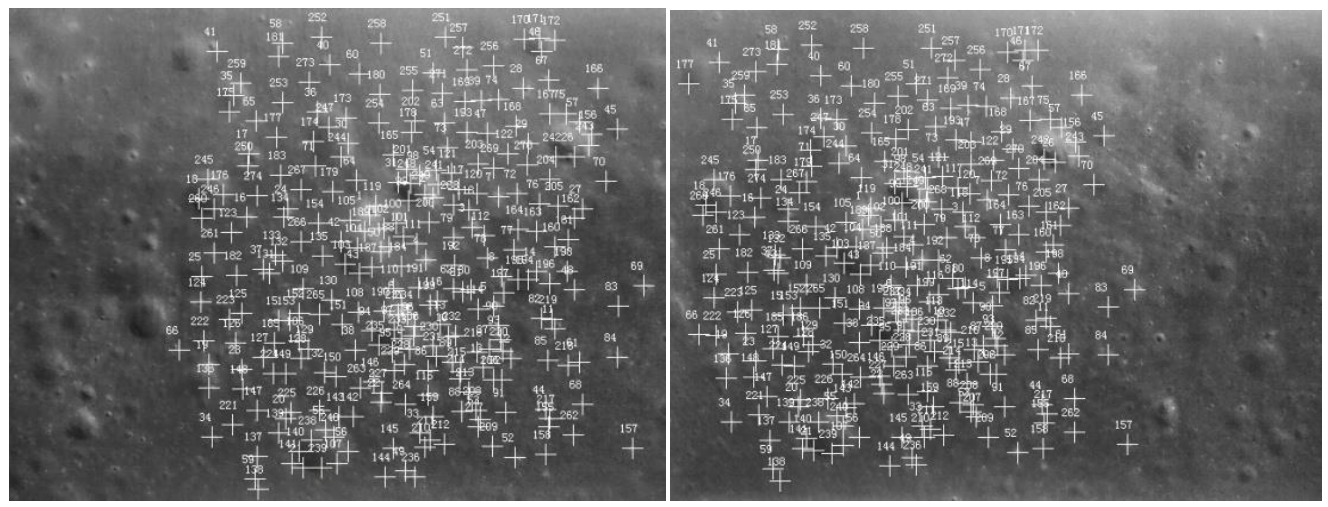

(c) The matching result of dense points for image feature points

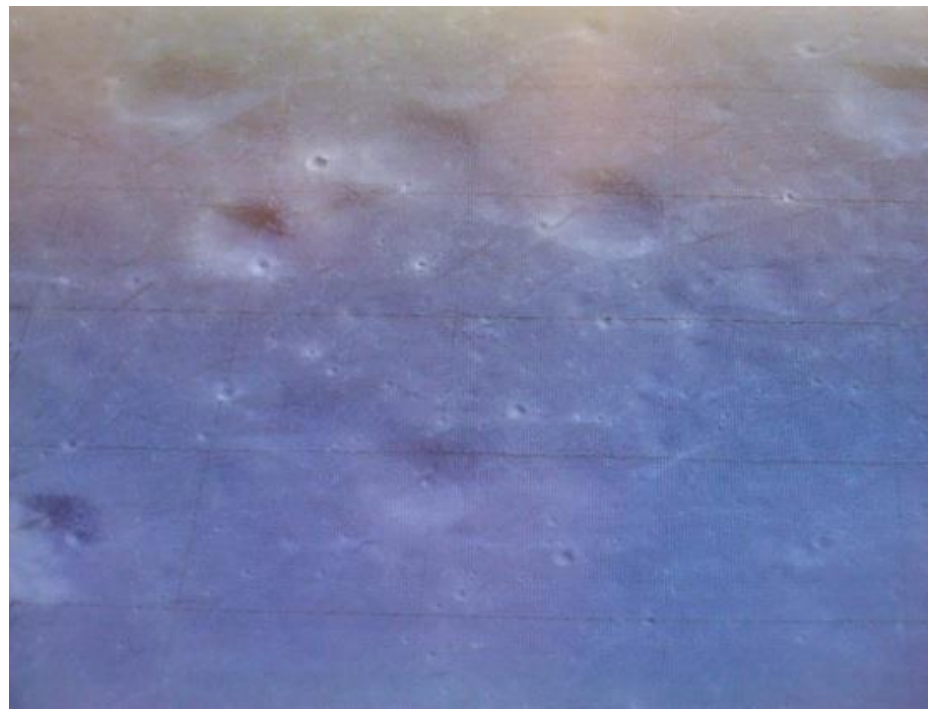

(d) The triangle grid of terrain reconstruction result's elevation data

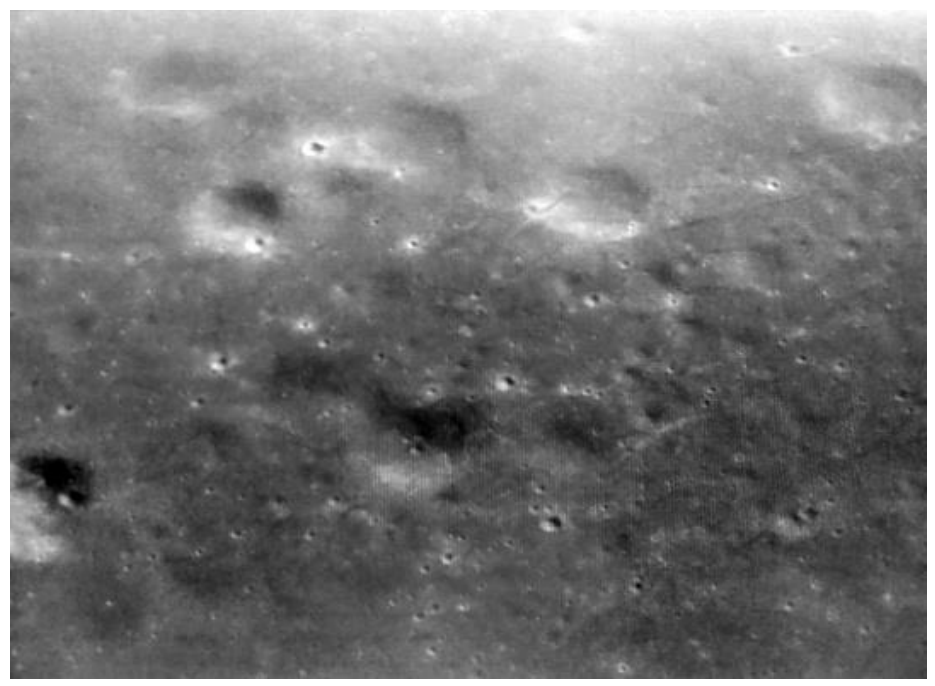

(e) The visualization of terrain reconstruction result's elevation data and texture data Figure 3. Terrain Reconstruction of detector ChangE III's Landing Camera 


\section{Conclusions}

In Deep Space Exploration, the acquired data contains many sequence landform images. By three-dimensional reconstruction technology, terrain information can be extracted from sequence images, such as elevation data and texture data. This paper focuses on adaptive feature points matching algorithm and adaptive three-dimensional reconstruction algorithm. To help detector ChangE III's landing field selection, adaptive terrain reconstruction algorithm is applied into the experimental image of landing camera. In future, the landing filed selection algorithm will be discussed on above research, so as to ensure the safety of detector landing.

\section{Acknowledgement}

This paper is a revised and expanded version of a paper entitled Research on Terrain Reconstruction of Twin Sequence Images in Lunar Exploration presented at The 2nd International Conference on Advanced Information and Computer Technology (AICT 2014), Budapest, Hungary and August 14-17, 2014.

This study is funded by the National Natural Science Foundation of China under Grant No.60873208.

\section{References}

[1] S. T. Barnard and M. A. Fischler, "Computational Stereo", ACM Computing Surveys, vol. 14, no. 553, (1982).

[2] U. R. Dhond and J. K. Aggarwal, "Structure from Stereo-A Review", IEEE Trans. Systems, Man, and Cybernetics, vol. 19, no. 1489, (1989).

[3] M. Z. Brown, D. Burschka and G. D. Hager, "Advances in Computational Stereo", IEEE Transactions on Pattern Analysis and Machine Intelligence, vol. 25, no. 993, (2003).

[4] S. D, SzeliskiR, "A Taxonomy and Evaluation of Dense two-Frame Stereo Correspondence Algorithms", International Journey of Computer Vision, vol. 47, no. 7, (2002).

[5] C. Schmid, A. Zisserman and A. Fitzgibbon, "Automatic Line Matching and 3DReconstruction of Buildings from Multiple Views", ISPRS Conference on Automatic Extraction of GIS Objects from Digital Imagery, IAPRS, vol. 32, no. 69, (1999).

[6] C. Rother and S. Carlsson, "Linear Multi View Reconstruction and Camera Recovery", Proc. 8thInternational Conference on Computer Vision, Vancouver, Canada, vol. 42, (2001).

[7] R. Hartley and A. Zisserman, "Multiple View Geometry in Computer Vision", Cambridge University Press, Cambridge, (2000).

[8] O. Faugeras and Q. T. Luong, "The Geometry of Multiple Images", The MIT Press, (2001).

[9] E. N. Mortensen, H. Deng and L. Shapiro, "A SIFT Descriptor with Global Context", Proceedings of International Conference on Computer Vision and Pattern Recognition, San Diego, USA, vol. 184, (2005).

[10] Z. Wenbin and Z. Zhengxu, "Research on Engineering Software Data Formats Conversion Network", Journal of Software, vol. 7, no. 2606, (2012).

[11] Z. Zhengxu and Z. W.enbin, "Engineering data formats: Visualization, conversion and migration", Intelligent Control and Information Processing (ICICIP), vol. 1, no. 295, (2011).

[12] S. Se, D. G. Lowe and J. J. Little, "Vision-Based Global Localization and Mapping for Mobile Robots", IEEE Transactions on Robotics, vol. 31, no. 364, (2005).

[13] Z. Liang, L. Jun and X. Sheng-Jun, "Research on a New Three-dimension Reconstruction and Visualization Technology of Concrete Based on CT Images", IJHIT, vol. 6, no. 5, (2013). 